\title{
Average degree conditions forcing a minor*
}

\author{
Daniel J. Harvey David R. Wood \\ School of Mathematical Sciences \\ Monash University \\ Melbourne, Australia \\ daniel.harvey87@gmail.com, david.wood@monash.edu \\ Submitted: Jun 11, 2015; Accepted: Feb 22, 2016; Published: Mar 4, 2016 \\ Mathematics Subject Classifications: $05 \mathrm{C} 83$
}

\begin{abstract}
Mader first proved that high average degree forces a given graph as a minor. Often motivated by Hadwiger's Conjecture, much research has focused on the average degree required to force a complete graph as a minor. Subsequently, various authors have considered the average degree required to force an arbitrary graph $H$ as a minor. Here, we strengthen (under certain conditions) a recent result by Reed and Wood, giving better bounds on the average degree required to force an $H$-minor when $H$ is a sparse graph with many high degree vertices. This solves an open problem of Reed and Wood, and also generalises (to within a constant factor) known results when $H$ is an unbalanced complete bipartite graph.
\end{abstract}

\section{Introduction}

Mader $[13,14]$ first proved that high average degree forces a given graph as a minor ${ }^{1}$. In particular, Mader $[13,14]$ proved that the following function is well-defined, where $d(G)$ denotes the average degree of a graph $G$ :

$$
f(H):=\inf \{D \in \mathbb{R}: \text { every graph } G \text { with } d(G) \geqslant D \text { contains an } H \text {-minor }\} .
$$

Often motivated by Hadwiger's Conjecture (see [18]), much research has focused on $f\left(K_{t}\right)$ where $K_{t}$ is the complete graph on $t$ vertices. For $3 \leqslant t \leqslant 9$, exact bounds on the number of edges due to Mader [14], Jørgensen [6], and Song and Thomas [19] imply that $f\left(K_{t}\right)=2 t-4$. But this result does not hold for large $t$. In particular, $f\left(K_{t}\right) \in \Theta(t \sqrt{\ln t})$, where the lower bound is independently by Kostochka [7, 8] and de la Vega [4] (based on

\footnotetext{
*Research supported by the Australian Research Council.

${ }^{1} \mathrm{~A}$ graph $H$ is a minor of a graph $G$ if a graph isomorphic to $H$ can be constructed from $G$ by vertex deletion, edge deletion and edge contraction.
} 
the work of Bollobás et al. [1]), and the upper bound is independently by Kostochka [7, 8] and Thomason [20]. Later, Thomason [21] determined the exact asymptotic constant.

Subsequently, other authors have considered $f(H)$ for arbitrary graphs $H$. Thomason [22] surveys some of these results. Myers and Thomason [16] determined an upper bound on $f(H)$ for all $H$, which is tight up to lower order terms when $H$ is dense. Hence much recent work has focused on $f(H)$ for sparse graphs $H$. There have been two major approaches in this case.

\section{Specific Sparse Graphs}

The first approach is to consider specific sparse graphs $H$. For example, Myers [15] considered unbalanced complete bipartite graphs $K_{s, t}$ where $s \ll t$. It is easily seen that $f\left(K_{1, t}\right)=t-1$. Myers proved that $f\left(K_{2, t}\right)=t+1$ for large $t$, and Chudnovsky et al. [3] proved the same result for all $t$. Kostochka and Prince [10] proved that $f\left(K_{3, t}\right)=t+3$ for large $t$. In fact, for all these results, the authors determined the exact maximum number of edges in a $K_{s, t}$-minor-free graph (for $s \leqslant 3$ ).

Myers conjectured that $f\left(K_{s, t}\right) \leqslant c_{s} t$ for some constant $c_{s}$ depending only on $s$. Strengthenings of this conjecture were independently proved by Kühn and Osthus [12] and Kostochka and Prince [9]. In particular, Kühn and Osthus [12] proved that for every $\epsilon \in\left(0,10^{-16}\right)$, if $t$ is sufficiently large (with respect to $\epsilon$ ) and $s \leqslant \epsilon^{6} \frac{t}{\ln t}$, then

$$
f\left(K_{s, t}\right) \leqslant(1+\epsilon) t
$$

Kostochka and Prince [9] proved a sharper bound on $f\left(K_{s, t}\right)$ under a stronger assumption: if $t>\left(180 s \log _{2} s\right)^{1+6 s \log _{2} s}$ then

$$
t+3 s-5 \sqrt{s} \leqslant f\left(K_{s, t}\right) \leqslant t+3 s .
$$

Later, Kostochka and Prince [11] proved an upper bound between (1) and (2) under a similar assumption to (1): if $s \leqslant \frac{t}{1000 \log _{2} t}$ then

$$
f\left(K_{s, t}\right) \leqslant t+8 s \log _{2} s .
$$

\section{General Sparse Graphs}

A second approach for sparse graphs is to determine upper bounds on $f(H)$ in terms of invariants of $H$. This is the approach of a recent paper by Reed and Wood [17]. Their main result is as follows: for every $t$-vertex graph $H$ with average degree $d(H)$ at least some constant $d_{0}$, then

$$
f(H) \leqslant 3.895 t \sqrt{\ln d(H)},
$$

If $d(H)$ is very small, then this result is not applicable. For all $H$, Reed and Wood [17] proved that

$$
f(H) \leqslant(1+3.146 d(H)) t .
$$

Inequalities (4) and (5) imply that for some constant $c$, for every $t$-vertex graph $H$,

$$
f(H) \leqslant c t \sqrt{\ln (d(H)+2)}
$$


A lower bound of Myers and Thomason [16] shows that (6) is tight for random or close-toregular graphs $H$. But for some graphs it is not tight. For example, for $K_{s, t}$ with $s \ll t$, inequality (6) says that $f\left(K_{s, t}\right) \leqslant c t \sqrt{\ln s}$ since $d\left(K_{s, t}\right) \approx 2 s$, whereas $f\left(K_{s, t}\right)=\Theta(t)$ as discussed above.

\section{Our Results}

In this paper, we make a first attempt at unifying these two approaches, both improving the bounds of Reed and Wood [17] in certain cases, and generalising the above mentioned results on unbalanced complete bipartite graphs up to a constant factor.

Theorem 1. There is a constant $d_{0}$ such that for integers $s \geqslant 0$ and $t \geqslant 3$ with $s \leqslant$ $10^{-5} \frac{t}{\ln t}$, for every $(s+t)$-vertex graph $H$ and set $S$ of $s$ vertices in $H$ such that $d(H-S) \geqslant$ $d_{0}$,

$$
f(H) \leqslant 3.895 t \sqrt{\ln d(H-S)} .
$$

Theorem 1 solves the second open problem of Reed and Wood [17], and is an improvement over (4) when $H$ contains a set $S$ of vertices with high degree (compared to the average degree). Then Theorem 1 forces $H$ as a minor in a graph $G$ as long as $d(G)>3.895 t \sqrt{\ln d}$, where $d$ is the average degree of $H-S$, instead of $H$ itself. Since vertices in $S$ have high degree, one expects that $d(H-S)$ is significantly less than $d(H)$.

Our second contribution relates $f(H)$ directly to $f(H-S)$.

Theorem 2. For $\epsilon \in(0,1]$ and integers $s \geqslant 0$ and $t \geqslant 3$ with $s \leqslant \frac{\epsilon}{100} \frac{t}{\ln t}$, for every graph $H$ and set $S$ of $s$ vertices in $H$,

$$
f(H) \leqslant 4\lceil(1+\epsilon) f(H-S)\rceil .
$$

Theorem 2 may allow a better result than Theorem 1 if we happen to know a good upper bound on $f(H-S)$. It also makes no explicit assumption on $d(H-S)$. Theorems 1 and 2 together imply the following general result.

Theorem 3. There is a constant $c$ such that for integers $s \geqslant 0$ and $t \geqslant 3$ with $s \leqslant 10^{-5} \frac{t}{\ln t}$, for every $(s+t)$-vertex graph $H$ and set $S$ of vertices in $H$ of size $s$,

$$
f(H) \leqslant c t \sqrt{\ln (d(H-S)+2)} .
$$

Consider Theorem 3 with $H=K_{s, t}$, where $S$ is the smaller colour class. Thus $d(H-$ $S)=0$ and Theorem 3 says that $f(H) \leqslant c t$, for some constant $c$ independent of $s$, which is within a constant factor of the bounds in (1), (2) and (3). Note however that these older results are still stronger, since Theorem 3 has a large multiplicative constant. On the other hand, Theorem 3 applies more generally when $H-S$ is not an independent set.

The following section contains a few preliminary lemmas. Section 3 contains proofs of Theorems 1, 2 and 3. Section 4 contains an observation about $f(H)$ when $H$ is seriesparallel. Section 5 considers some future directions. 


\section{Preliminaries}

We first prove a few useful preliminaries before proving our theorems.

A connected dominating set $A$ of a graph $G$ is a set of vertices such that the induced subgraph $G[A]$ is connected, and each vertex of $V(G)$ is either in $A$ or adjacent to a vertex in $A$. Lemma 4 is well known, and weaker than other previous results such as that by Caro et al. [2]. We present it here for completeness and because the upper bound on the order of the connected dominating set has no lower order terms, which simplifies the calculations in Section 3. Similarly, Lemma 5 resembles a previous result of Kostochka and Prince [9].

Lemma 4. Every graph $G$ with $n$ vertices and minimum degree at least $\frac{1}{2} n$ has a connected dominating set of order less than $2 \log _{2} n$.

Proof. Let $A$ be a set of $\left\lfloor\log _{2} n\right\rfloor$ vertices in $G$ chosen uniformly at random. For each vertex $x$ of $G-A$, since $\operatorname{deg}_{G}(x) \geqslant \frac{n}{2}$, the probability that $x$ has no neighbour in $A$ is less than $\left(\frac{1}{2}\right)^{\log _{2} n}=\frac{1}{n}$, and so the probability that $A$ does not dominate $G$ is less than 1. Hence there is some choice of $A$ that does dominate $G$. Label the vertices of this $A$ by $x_{1}, \ldots, x_{\left\lfloor\log _{2} n\right\rfloor}$. For $i=1, \ldots,\left\lfloor\log _{2} n\right\rfloor-1$, let $v_{i}=x_{i+1}$ if $x_{i}$ and $x_{i+1}$ are adjacent, otherwise let $v_{i}$ be a common neighbour of $x_{i}$ and $x_{i+1}$, which exists since $x_{i}$ and $x_{i+1}$ each have at least $\frac{n}{2}$ neighbours in a set of $n-2$ vertices. Then $A \cup\left\{v_{1}, \ldots, v_{\left\lfloor\log _{2} n\right\rfloor-1}\right\}$ is a connected dominating set of $G$ with less than $2 \log _{2} n$ vertices.

Lemma 5. For every integer $s \geqslant 1$ and $\rho \geqslant \frac{1}{2}$, every graph $G$ with $n$ vertices and minimum degree at least $\rho n+2 s \log _{2} n$ contains vertex sets $A_{1}, \ldots, A_{s}$ and subgraphs $G_{0}, G_{1}, \ldots, G_{s}$ such that $G_{0}=G, G_{i}=G-\left(A_{1} \cup \cdots \cup A_{i}\right)$ for $i \in\{1, \ldots, s\}$, and

(a) $A_{i}$ is a connected dominating set in $G_{i-1}$,

(b) $\left|A_{i}\right|<2 \log _{2} n$,

(c) $G_{i}$ has minimum degree at least $\rho n+2(s-i) \log _{2} n$.

(d) $G_{i}$ has at least $n-2 i \log _{2} n$ vertices.

Proof. We use induction on $i$. Say $i=1$. Let $A_{1}$ be a connected dominating set in $G_{0}=G$ from Lemma 4. This satisfies $(a)$ and $(b)$. The minimum degree of $G_{1}=G-A_{1}$ is at least the minimum degree of $G$ minus $\left|A_{1}\right|$, which proves $(c)$. The number of vertices of $G_{1}$ is at least $n-\left|A_{1}\right|$, which proves $(d)$.

Assume our lemma holds for $i-1$. Let $A_{i}$ be a connected dominating set in $G_{i-1}$ from Lemma 4 ; such a set exists since $G_{i-1}$ has minimum degree greater than $\rho n \geqslant \frac{1}{2} n$. This satisfies $(a)$ and $(b)$. Finally, $G_{i}$ has minimum degree at least $\rho n+2(s-(i-1)) \log _{2} n-$ $\left|A_{i}\right|>\rho n-2(s-i) \log _{2} n$ and $\left|V\left(G_{i}\right)\right| \geqslant n-2(i-1) \log _{2} n-\left|A_{i}\right|>n-2 i \log _{2} n$, as required.

Finally, we cite two key results of Reed and Wood [17]. 
Lemma 6 (Reed and Wood [17], Lemma 2.5). For every integer $k \geqslant 1$, every graph with average degree at least $4 k$ contains a complete graph $K_{k}$ as a minor or contains a minor with $n$ vertices and minimum degree $\delta$, where $\delta \geqslant 0.6518 n$, and $k \leqslant \delta<n \leqslant 4 k$.

Lemma 7 (Reed and Wood [17], Lemma 5.1). For all $\lambda \in\left(\frac{1}{2}, 1\right)$ and $\epsilon \in(0, \lambda)$ there exists $d_{0}(\lambda, \epsilon)$ such that for every graph $H$ with $t$ vertices and average degree $d \geqslant d_{0}$ every graph $G$ with $n \geqslant(1+\epsilon)\left\lceil\sqrt{\log _{b} d}\right\rceil t$ vertices and minimum degree at least $\lambda n$ contains $H$ as a minor, where $b=(1-\lambda+\epsilon)^{-1}$.

\section{Proofs of Theorems}

Proof of Theorem 1. Let $G$ be a graph with $d(G) \geqslant 3.895 t \sqrt{\ln d}$ where $d:=d(H-S) \geqslant$ $d_{0}$. Let $k:=\left\lfloor\frac{1}{4}(3.895 t \sqrt{\ln d})\right\rfloor$. Our goal is to show that $G$ contains an $H$-minor. We can take $d_{0}$ large enough so that $k \geqslant 2 t>1$. By Lemma $6, G$ contains either $K_{2 t}$ or $G^{\prime}$ as a minor, where $G^{\prime}$ is a graph with $n$ vertices and minimum degree at least $0.6518 n$ such that $k+1 \leqslant n \leqslant 3.895 t \sqrt{\ln d}$. If $G$ contains a $K_{2 t}$ minor, then $G$ contain a $K_{s+t}$ minor and we are done. Otherwise apply Lemma 5 to $G^{\prime}$ where $\rho=0.6517$. Since $s \leqslant 10^{-5} \frac{t}{\ln t}<5 \times 10^{-5} \frac{t}{\log _{2} t} \leqslant 5 \times 10^{-5} \frac{n}{\log _{2} n}$, we have $2 s \log _{2} n \leqslant 10^{-4} n$, and it follows that $G^{\prime}$ has minimum degree at least $\rho n+2 s \log _{2} n$, as required. Let $G^{\prime \prime}:=G_{s}$ from Lemma 5. Then $G^{\prime \prime}$ has minimum degree at least $0.6517 n>0.6517\left|V\left(G^{\prime \prime}\right)\right|$.

We wish to find an $(H-S)$-minor of $G^{\prime \prime}$. Then contracting each $A_{i}$ to a single vertex gives an $H$-minor in $G^{\prime}$. We now verify that Lemma 7 gives the desired $(H-S)$-minor in $G^{\prime \prime}$, where $\lambda:=0.6517$ and $\epsilon:=10^{-6}$ and $b:=(1-\lambda+\epsilon)^{-1}$. Clearly $G^{\prime \prime}$ has sufficient minimum degree (by our choice of $\lambda$ ) and the average degree $d$ of $H-S$ is sufficiently large (since we may assume $d_{0}$ is sufficiently large in terms of absolute constants), and so all that remains is to ensure that $G^{\prime \prime}$ has sufficiently many vertices. Note $\left|V\left(G^{\prime \prime}\right)\right| \geqslant n-2 s \log _{2} n$ from Lemma $5(\mathrm{~d})$. We may choose $d_{0}$ large enough so that $\frac{3.895 t \sqrt{\ln d}}{\log _{2}(3.895 t \sqrt{\ln d})} \geqslant 100 \frac{t}{\ln t}$ and so $s \leqslant 10^{-5} \frac{t}{\ln t} \leqslant 10^{-7} \frac{3.895 t \sqrt{\ln d}}{\log _{2}(3.895 t \sqrt{\ln d})}$. Thus it follows that

$$
\begin{aligned}
\left|V\left(G^{\prime \prime}\right)\right| & \geqslant n-2\left(10^{-7}\right) \frac{3.895 t \sqrt{\ln d}}{\log _{2}(3.895 t \sqrt{\ln d})} \log _{2} n \\
& \geqslant\left\lfloor\frac{1}{4}(3.895 t \sqrt{\ln d})\right\rfloor+1-2\left(10^{-7}\right)(3.895 t \sqrt{\ln d}) \\
& \geqslant \frac{1}{4}(3.895 t \sqrt{\ln d})-2\left(10^{-7}\right)(3.895 t \sqrt{\ln d}) \\
& =\left(\frac{1}{4}-2\left(10^{-7}\right)\right)(3.895 t \sqrt{\ln d}) \\
& =\left(\frac{1}{4}-2\left(10^{-7}\right)\right)\left(3.895 t \sqrt{\ln b} \sqrt{\log _{b} d}\right) \\
& \geqslant 1.00002 \sqrt{\log _{b} d} t \\
& =(1+20 \epsilon) \sqrt{\log _{b} d} t \\
& \geqslant(1+\epsilon)\left(1+\sqrt{\log _{b} d}\right) t \quad \text { (taking d large enough) } \\
& \geqslant(1+\epsilon)\left\lceil\sqrt{\log _{b} d}\right\rceil t .
\end{aligned}
$$

Hence it follows that $G^{\prime \prime}$ contains an $(H-S)$-minor. This is an $(H-S)$-minor in $G^{\prime}$ that avoids the sets $A_{1}, \ldots, A_{s}$. Hence $G^{\prime}$ (and also $G$ ) contains our desired $H$-minor. 
Proof of Theorem 2. Let $G$ be a graph with average degree $d(G) \geqslant 4 k$, where $k:=$ $\lceil(1+\epsilon) f(H-S)\rceil$. If $s=0$ this result is trivial, so assume $s \geqslant 1$. It follows from Lemma 6 that $G$ contains either a $K_{k}$-minor or a minor $G^{\prime}$ with $n$ vertices and minimum degree $\delta\left(G^{\prime}\right) \geqslant 0.6518 n$ such that $k \leqslant \delta\left(G^{\prime}\right)<n \leqslant 4 k$. Since $f(H-S) \geqslant t-2$ (as $K_{t-1}$ has no $(H-S)$-minor $)$, it follows that $k \geqslant(1+\epsilon) f(H-S) \geqslant(1+\epsilon)(t-2) \geqslant$ $t-2+100 s \ln t-2 \epsilon \geqslant t+100 s-4>t+s$. Hence a $K_{k}$-minor contains an $H$-minor. Now assume Lemma 6 finds $G^{\prime}$ as a minor.

We wish to apply Lemma 5 to $G^{\prime}$ with $\rho=\frac{1}{2}$. It is sufficient to show that $0.1518 n \geqslant$ $2 s \log _{2} n$. Since $n \geqslant k+1 \geqslant(1+\epsilon) f(H-S)+1 \geqslant t-1+\epsilon f(H-S)$, and $n$ and $t$ are integers, it follows that $n \geqslant t$. Thus $s \leqslant \frac{\epsilon}{100} \frac{t}{\ln t} \leqslant \frac{\epsilon}{100} \frac{n}{\ln n}$ and so $2 s \log _{2} n \leqslant \frac{n}{50 \ln 2}<0.1518 n$ as required. Let $G^{\prime \prime}:=G_{s}$ from Lemma 5. By Lemma 5(b), it follows that $G^{\prime \prime}$ has minimum degree at least $\delta\left(G^{\prime}\right)-2 s \log _{2} n \geqslant k-2 s \log _{2} n$. From our upper bound on $s$ and since $k<n \leqslant 4 k$, it follows that $k-2 s \log _{2} n \geqslant k-\epsilon \frac{n}{50 \ln n} \log _{2} n \geqslant k\left(1-\frac{2}{25 \ln 2} \epsilon\right) \geqslant$ $(1+\epsilon)(1-0.1155 \epsilon) f(H-S)>f(H-S)$. Thus $d\left(G^{\prime \prime}\right)>f(H-S)$ and $G^{\prime \prime}$ contains an $(H-S)$-minor. Contracting the sets $A_{1}, \ldots, A_{s}$ to single vertices gives an $H$-minor in $G^{\prime}$, and thus also in $G$.

Proof of Theorem 3. First suppose that $d(H-S) \geqslant d_{0}$, where $d_{0}$ is from Theorem 1 . Let $c \geqslant 3.895$. By Theorem $1, f(H) \leqslant 3.895 t \sqrt{\ln d(H-S)} \leqslant c t \sqrt{\ln (d(H-S)+2)}$ as required. Alternatively, $d(H-S)<d_{0}$. By (5) we have $f(H-S) \leqslant(1+$ $3.146 d(H-S)) t \leqslant\left(1+3.146 d_{0}\right) t$. Let $\epsilon=10^{-3}$. It follows from Theorem 2 that $f(H) \leqslant 4\left\lceil(1+\epsilon)\left(1+3.146 d_{0}\right) t\right\rceil$. Setting $c$ large enough, this proves $f(H) \leqslant$ $c t \sqrt{\ln (d(H-S)+2)}$, as required.

\section{Series-Parallel Graphs}

A graph is series-parallel if it contains no $K_{4}$ minor (or equivalently, it has treewidth at most 2). Reed and Wood [17, Lemma 3.3] proved that $f(H) \leqslant 6.929 t$ for every $t$ vertex 2-degenerate graph $H$. Every series-parallel graph $H$ is 2-degenerate, implying $f(H) \leqslant 6.929 t$. Here we make the following improvement.

Proposition 8. For every t-vertex series-parallel graph $H$,

$$
f(H) \leqslant 2 t-4 \text {. }
$$

Proof. A 2-tree is a graph that can be constructed by starting with $K_{3}$ and repeatedly selecting an edge and adding a new vertex adjacent to exactly the endpoints of that edge. It is well known that 2-trees are exactly the edge-maximal series-parallel graphs [5]. Hence it suffices to prove that $f(H) \leqslant 2 t-4$ for every $t$-vertex 2 -tree $H$. Say a graph $G$ is minor-minimal (with respect to $t$ ) if $d(G) \geqslant 2 t-4$ but every proper minor of $G$ has average degree less than $2 t-4$. Considering the effect of contracting an edge on the average degree, it is easily seen that every edge of a minor-minimal graph is in at least $t-2$ triangles; see [17, Lemma 2.1]. It suffices to prove that every minor-minimal graph $G$ contains an $H$-minor. In fact, we prove that every 2 -tree $H_{0}$ on at most $t$ vertices can be embedded in $G$ as a subgraph. 
We do this by induction on $\left|V\left(H_{0}\right)\right|$ with $G$ fixed. Suppose $\left|V\left(H_{0}\right)\right|=3$. Then every edge of $G$ is in at least $t-2 \geqslant 1$ triangles, and it is trivial to embed $H_{0}$. Now suppose $\left|V\left(H_{0}\right)\right|=i>3$. There is a vertex $v \in V\left(H_{0}\right)$ with neighbours $x$ and $y$ such that $H_{0}-v$ is also a 2-tree. By induction, $H_{0}-v$ can be embedded in $G$. Let $x^{\prime}, y^{\prime}$ denote the vertices of $G$ where $x, y$ are respectively embedded. Then $x^{\prime} y^{\prime}$ is an edge of $G$ in at least $t-2 \geqslant\left|V\left(H_{0}\right)-\{v, x, y\}\right|+1$ triangles. Hence there exists a common neighbour $w$ of $x^{\prime}$ and $y^{\prime}$ in $G$ where no vertex of $H_{0}-\{v, x, y\}$ is embedded. Clearly, neither $x$ nor $y$ are embedded at $w$. Embedding $v$ at $w$, we obtain $H_{0}$ as a subgraph of $G$, as required.

\section{Open Problems}

An obvious question is whether the upper bound on $f(H)$ provided by Theorem 3 is within a constant factor of optimal for all $H$. If $S$ is a small (perhaps empty) set of vertices in $H$ and $H-S$ is sufficiently large and either random or close-to-regular, then it follows from the lower bound of Myers and Thomason [16] that

$$
f(H) \geqslant f(H-S) \geqslant c|V(H-S)| \sqrt{\ln (d(H-S))},
$$

and Theorem 3 is within a constant factor of optimal.

Is it possible that Theorem 3 always gives a result within a constant factor of optimal (for the best possible choice of $S$ )? If not, for which graphs is Theorem 3 not within a constant factor of optimal, and what approach should we take for such graphs? More generally, is there a small set of upper bounds on $f(H)$ such that for each graph $H$, one of these bounds is within a constant factor of optimal? Similarly, is there a constant factor approximation algorithm for computing $f(H)$ ?

\section{References}

[1] Béla Bollobás, Paul A. Catlin, and Paul Erdős. Hadwiger's conjecture is true for almost every graph. European J. Combin., 1(3):195-199, 1980. doi:10.1016/S0195-6698(80)80001-1.

[2] Yair Caro, Douglas B. West, and Raphael Yuster. Connected domination and spanning trees with many leaves. SIAM J. Discrete Math., 13(2):202-211, 2000. doi:10.1137/S0895480199353780.

[3] Maria Chudnovsky, Bruce A. Reed, and Paul Seymour. The edgedensity for $K_{2, t}$ minors. J. Combin. Theory Ser. B, 101(1):18-46, 2011. doi:10.1016/j.jctb.2010.09.001.

[4] W. Fernandez DE LA VEGA. On the maximum density of graphs which have no subcontraction to $K^{s}$. Discrete Math., 46(1):109-110, 1983. doi : 10.1016/0012-365X (83)90280-7.

[5] Reinhard Diestel. Graph theory, vol. 173 of Graduate Texts in Mathematics. Springer-Verlag, Berlin, 4th edn., 2010. http://diestel-graph-theory.com/.

[6] Leif K. Jørgensen. Contractions to $K_{8}$. J. Graph Theory, 18(5):431-448, 1994. doi:10.1002/jgt.3190180502. 
[7] Alexandr V. Kostochka. The minimum Hadwiger number for graphs with a given mean degree of vertices. Metody Diskret. Analiz., 38(38):37-58, 1982.

[8] Alexandr V. Kostochka. Lower bound of the Hadwiger number of graphs by their average degree. Combinatorica, 4(4):307-316, 1984. doi:10.1007/BF02579141.

[9] Alexandr V. Kostochka and Noah Prince. On $K_{s, t}$-minors in graphs with given average degree. Discrete Math., 308(19):4435-4445, 2008. doi:10.1016/j.disc.2007.08.041.

[10] Alexandr V. Kostochka and Noah Prince. Dense graphs have $K_{3, t}$ minors. Discrete Math., 310(20):2637-2654, 2010. doi:10.1016/j.disc.2010.03.026.

[11] Alexandr V. Kostochka and Noah Prince. On $K_{s, t}$-minors in graphs with given average degree, II. Discrete Math., 312(24):3517-3522, 2012. doi:10.1016/j.disc.2012.08.004.

[12] Daniela Kühn And Deryk Osthus. Forcing unbalanced complete bipartite minors. European J. Combin., 26(1):75-81, 2005. doi:10.1016/j.ejc.2004.02.002.

[13] Wolfgang Mader. Homomorphieeigenschaften und mittlere Kantendichte von Graphen. Mathematische Annalen, 174:265-268, 1967. doi:10.1007/BF01364272.

[14] Wolfgang Mader. Homomorphiesätze für Graphen. Math. Ann., 178:154-168, 1968. doi:10.1007/BF01350657.

[15] Joseph Samuel Myers. The extremal function for unbalanced bipartite minors. Discrete Math., 271(1-3):209-222, 2003. doi:10.1016/S0012-365X(03)00051-7.

[16] Joseph Samuel Myers And Andrew Thomason. The extremal function for noncomplete minors. Combinatorica, 25(6):725-753, 2005. doi:10.1007/s00493-005-0044-0.

[17] Bruce A. Reed and David R. Wood. Forcing a sparse minor. Combin. Probab. Comput., 25:300-322, 2016. doi:10.1017/S0963548315000073.

[18] Paul D. Seymour. Hadwiger's conjecture, 2015. https://web.math.princeton. edu/ pds/papers/hadwiger/paper.pdf.

[19] Zi-XiA Song And Robin Thomas. The extremal function for $K_{9}$ minors. $J$. Combin. Theory Ser. B, 96(2):240-252, 2006. doi:10.1016/j.jctb.2005.07.008.

[20] Andrew Thomason. An extremal function for contractions of graphs. Math. Proc. Cambridge Philos. Soc., 95(2):261-265, 1984. doi:10.1017/S0305004100061521.

[21] Andrew Thomason. The extremal function for complete minors. J. Combin. Theory Ser. B, 81(2):318-338, 2001. doi:10.1006/jctb.2000.2013.

[22] Andrew Thomason. Extremal functions for graph minors. In More Sets, Graphs and Numbers, vol. 15 of Bolyai Society Mathematical Studies, pp. 359-380. 2006. doi:10.1007/978-3-540-32439-3_17. 